\title{
Analysis on clinical distribution and drug resistance of Klebsiella pneumoniae isolated for 5 consecutive years
}

\author{
Yueyu Li, Hongbo Guo* \\ Clinical Laboratory, Baogang Hospital, Baotou, Inner Mongolia, China
}

Received: October 9, 2018

DOI: $10.14725 /$ dcc.v5n4p36
Accepted: November 27, $2018 \quad$ Online Published: December 10, 2018

URL: http://dx.doi.org/10.14725/dcc.v5n4p36

\begin{abstract}
Objective: To analyze the clinical distribution and drug resistance of Klebsiella pneumoniae isolated from patients in a certain hospital and provide a basis for the rational use of antibiotics in the clinical treatment for the infection of Klebsiella pneumoniae.

Methods: 1,192 strains of Klebsiella pneumoniae isolated from clinical specimens from 2012 to 2016 were collected. The strains were identified by VITEK-2 Compact Microbiological Identification System, and the corresponding results of the antimicrobial susceptibility test were interpreted in accordance with the standards recommended by Clinical and Laboratory Standards Institute (CLSI).

Results: 1,192 strains of Klebsiella pneumoniae were mainly isolated from sputum (65.6\%), and most of them were from Respiratory Medicine Department and Medical Intensive Care Unit of Respiratory Medicine Department (MICU), accounting for $41.4 \%$. Out of 1,192 strains, 448 strains were detected to produce extended-spectrum beta-lactamases (ESBLs), accounting for $37.6 \%$. In addition, the detection rates of ESBL-producing Klebsiella pneumoniae for 5 consecutive years showed an increasing trend year by year, and they were higher than the national average values published by China Antimicrobial Resistance Surveillance System (CARSS) in the corresponding period. The drug resistance rate of ESBL-producing Klebsiella pneumoniae was significantly higher than that of non ESBL-producing strains.

Conclusions: The infection caused by Klebsiella pneumoniae mainly occurs in the lower respiratory tract, and the drug resistance rates of Klebsiella pneumoniae to antibiotics in the drug susceptibility spectrum are maintained at a high level. Therefore, the rational selection of antibiotics for the clinical treatment of lower respiratory tract infection caused by Klebsiella pneumoniae must be based on the production of ESBLs and the results of antimicrobial susceptibility test.
\end{abstract}

Key Words: Extended-spectrum beta-lactamases, Klebsiella pneumoniae, Drug resistance rate, Antibiotics

Klebsiella pneumoniae, a type of gram-negative bacilli, is an important pathogenic bacteria which is not only one of important conditions causing clinical infection and but also one of the common enterobacteriaceae bacteria causing nosocomial infection. ${ }^{[1,2]}$ It often leads to some diseases such as lower respiratory tract infection, wound in- fection, bacteremia and urinary tract infection etc. Its resistance mechanism is mainly associated with the production of extended-spectrum beta-lactamases (ESBLs) and amp cephalosporinase (AmpC) mediated by plasmids. As a result of the extensive use of broad-spectrum antibiotics in recent years, the infection and drug resistance caused by this China.

*Correspondence: Hongbo Guo; E-mail: guohongbobaotou@ 126.com; Address: Clinical Laboratory, Baogang Hospital, Baotou, Inner Mongolia, 
type of bacteria have been on rise year by year, and the proportion of ESBL-producing Klebsiella pneumoniae in Klebsiella pneumoniae has also been increasing. Therefore, in clinical practice, it is worthy to be noted that the rational selection of antibiotics should be based on the production of ESBLs and the results of antimicrobial susceptibility test. To understand the clinical distribution and drug resistance trend of Klebsiella pneumoniae in this hospital to provide a basis for the clinical development of reasonable, scientific and effective antimicrobial treatment options, the clinical distribution and drug resistance of Klebsiella pneumoniae isolated clinically in the hospital from 2012 to 2016 were analyzed and reported as follows.

\section{Materials and methods}

\subsection{Strain origin}

1,192 strains of Klebsiella pneumoniae isolated from sputum, secretions, whole blood, urine and other specimens sent by the hospital for clinical examinations from 2012 to 2016 were collected (excluding the repeated strains isolated from the same site in the same patient).

\subsection{The source of antibiotic discs and medium}

All antibiotic discs, Columbia blood agar plates, MacC agar plates and $\mathrm{M}-\mathrm{H}$ agar were purchased from Oxoid (UK), except that ceftazidime/clavulanic acid and cefotaxime/clavulanic acid were purchased from Wenzhou BioKont Biotechnology Co., Ltd.

\subsection{Strain identification and antimicrobial suscep- tibility test}

VITEK-2 Compact Microbiological Identification System made by BioMérieux (France) was applied to strain identification, and Kirby-Bauer disc agar diffusion method (K-B method) or MIC method of VITEK-2 Compact Microbiological Identification System was used to perform the antimicrobial susceptibility test. The results were interpreted in accordance with the standards established by Clinical and Laboratory Standards Institute (CLSI) in 2012 and its update versions.

\subsection{ESBLs screening and identification}

Inhibitor-potentiated disc diffusion test recommended by CLSI was applied to ESBLs disc screening and identification test. If one or both of the combinations of cefotaxime and cefotaxime/clavulanic acid, ceftazidime and ceftazidime/clavulanic acid met the requirements that the difference in the diameter of zone of inhibition was no less than
$5 \mathrm{~mm}$, these strains would be identified as ESBL-producing strains.

\subsection{Quality-control strains}

Quality-control strains were Escherichia coli ATCC25922 and Pseudomonas aeruginosa ATCC27853; and the qualitycontrol strains of ESBL disc screening and identification test were Escherichia coli ATCC25922 and Klebsiella pneumoniae ATCC700603, which were purchased from Clinical Laboratory Center of Ministry of Health.

\subsection{Statistical methods}

Whonet 5.6 software was applied to the statistical analysis of the drug resistance, excluding the repeated strains isolated from the same site in the same patient.

\section{Results}

\subsection{Specimen distribution}

From 2012 to 2016, 1,192 strains of Klebsiella pneumoniae were isolated from clinical specimens, including 782 strains from sputum (65.6\%), 139 strains from secretions (11.7\%), 135 strains from whole blood $(11.3 \%), 75$ strains from urine $(6.3 \%)$ and 61 strains from other specimens $(5.1 \%)$.

\subsection{Department distribution}

The top five departments whose detection rates of Klebsiella pneumoniae isolated from 2012 to 2016 ranked high were Respiratory Medicine Department, Medical Intensive Care Unit of Respiratory Medicine Department (MICU), ICU, Department of Burns and Department of Geriatrics, among which Respiratory Medicine Department and MICU were of the highest detection rates, accounting for $41.4 \%$ of the total number of Klebsiella pneumoniae (see Table 1).

\subsection{The detection rate of ESBL-producing Kleb- siella pneumoniae}

Among 1,192 strains of Klebsiella pneumoniae, 448 strains were ESBL-producing strains, accounting for $37.6 \%$. In addition, the detection rates of ESBL-producing Klebsiella pneumoniae for 5 consecutive years were $32.3 \%, 29.2 \%$, $42.2 \%, 43.6 \%$ and $43.5 \%$ respectively, showing an increasing trend year by year, and they were higher than the national average values published by China Antimicrobial Resistance Surveillance System (CARSS) in the same period. See Table 2 for the detection rates of ESBL-producing Klebsiella pneumoniae for 5 consecutive years. 
Table 1: Department distribution of Klebsiella pneumoniae

\begin{tabular}{lcc}
\hline Department & The number of strains isolated & Constituent ratio (\%) \\
\hline Respiratory Medicine Department & 312 & 26.2 \\
MICU & 181 & 15.2 \\
ICU & 134 & 9.2 \\
Department of Burns & 111 & 9.3 \\
Department of Geriatrics & 105 & 3.9 \\
Department of Urology Surgery & 41 & 3.4 \\
Department of Endocrinology & 61 & 5.1 \\
Department of Neurology & 68 & 5.7 \\
Department of General Surgery & 35 & 2.9 \\
Department of Oncology & 33 & 2.8 \\
Department of Hematology & 26 & 2.2 \\
Others & 85 & 7.1 \\
Total & 1,192 & 100.0 \\
\hline
\end{tabular}

Table 2: Detection rates of ESBL-producing Klebsiella pneumoniae from 2012 to 2016

\begin{tabular}{lccc}
\hline Year & The total number of strains & The number of ESBL-producing strains & Detection rate $(\%)$ \\
\hline 2012 & 235 & 76 & 32.3 \\
2013 & 291 & 85 & 29.2 \\
2014 & 225 & 95 & 42.2 \\
2015 & 234 & 102 & 43.6 \\
2016 & 207 & 90 & 43.5 \\
Total & 1,192 & 448 & 37.6 \\
\hline
\end{tabular}

Note. "The total number of strains" refers to the number of Klebsiella pneumoniae detected

\subsection{Drug resistance rates to common antibiotics}

The drug resistance rates of Klebsiella pneumoniae to piperacillin and compound sulfamethoxazole were $41.9 \%$ and $42.2 \%$. The drug resistance rates of the first, second and third generation cephalosporins (excluding ceftazidime) ranged from $40.0 \%$ to $45.2 \%$, except that the drug resistance rates to cefoxitin, ceftazidime and cefepime were less than $20 \%$. The drug resistance rates of Klebsiella pneumoniae to piperacillin/tazobactam, amikacin, imipenem and meropenem were all lower than $3.9 \%$. See Table 3 for the drug resistance rates of 1,192 strains of Klebsiella pneumoniae to common antibiotics.

\subsection{Drug resistance rates of ESBL-producing and non ESBL-producing Klebsiella pneumoniae to common antibiotics in the drug susceptibility spectrum}

After the analysis of the drug resistance rates of Klebsiella pneumoniae for 5 consecutive years, it was found that the drug resistance rates of ESBL-producing Klebsiella pneumoniae to various antibiotics in the drug susceptibil- ity spectrum (except imipenem, meropenem and amikacin) were significantly higher than those of non ESBL-producing Klebsiella pneumoniae (see Table 4).

\section{Discussion}

Klebsiella pneumoniae exists extensively in the microecological environment such as water and soil, and it is easy to be colonized on the respiratory tract and the skin surface in hospitalized patients. When the body immunity is low, it could cause a variety of site infections such as respiratory tract infection, skin and soft tissue infections and systemic infection. It has become one of the important pathogens causing nosocomial infection at present. ${ }^{[3-5]}$ The investigation results showed that Klebsiella pneumoniae isolated in a certain hospital were mainly from sputum, wound secretions and whole blood specimens, indicating that the lower respiratory tract, skin wounds and bacteremia are important parts of Klebsiella pneumoniae infection, especially the sputum specimens accounting for $65.6 \%$, which was similar to the data in the study made by Zhou $\mathrm{R}$ et al. ${ }^{[6]}$ on distribution and antibiotic resistance of 855 isolates of Klebsiella 
pneumoniae (68.9\%). From the perspective of department pecially the patients in MICU, which may be related to the distribution, Respiratory Medicine Department and MICU critical condition, the extensive use of antibiotics and invahad the highest detection rates, accounting for $41.4 \%$, es- sive operations such as the use of ventilator.

Table 3: Drug resistance rates of 1,192 strains of Klebsiella pneumoniae to common antibiotics (\%)

\begin{tabular}{|c|c|c|c|c|c|c|}
\hline Antibiotics & $\begin{array}{l}2012 \\
(n=235)\end{array}$ & $\begin{array}{l}2013 \\
(n=291)\end{array}$ & $\begin{array}{l}2014 \\
(n=225)\end{array}$ & $\begin{array}{l}2015 \\
(n=234)\end{array}$ & $\begin{array}{l}2016 \\
(n=207)\end{array}$ & $\begin{array}{l}\text { Total } \\
(\mathrm{n}=1,192)\end{array}$ \\
\hline Piperacillin & 35.5 & 31.6 & 44.9 & 46.2 & 46.4 & 41.9 \\
\hline Piperacillin/Tazobactam & 0.9 & 3.1 & 0.9 & 1.3 & 6.8 & 2.4 \\
\hline Cefazolin & 42.7 & 37.6 & 46.9 & 44.0 & 50.5 & 45.2 \\
\hline Cefuroxime & 38.4 & 36.3 & 43.9 & 44.4 & 47.6 & 43.2 \\
\hline Ceftazidime & 15.8 & 12.4 & 13.3 & 11.1 & 23.7 & 16.1 \\
\hline Ceftriaxone & 33.3 & 30.2 & 41.8 & 43.6 & 46.6 & 40.0 \\
\hline Cefotaxime & 33.3 & 29.2 & 42.2 & 43.6 & 43.5 & 40.0 \\
\hline Cefepime & 7.4 & 4.1 & 8.9 & 7.3 & 22.2 & 10.4 \\
\hline Cefoxitin & 9.4 & 13.5 & 7.1 & 13.6 & 8.7 & 8.5 \\
\hline Aztreonam & 15.9 & 11.3 & 14.8 & 0.0 & 20.8 & 18.2 \\
\hline Imipenem & 0.4 & 0.0 & 0.4 & 0.0 & 2.9 & 0.6 \\
\hline Meropenem & 0.4 & 0.0 & 0.4 & 0.0 & 2.9 & 0.6 \\
\hline Amikacin & 3.8 & 0.0 & 0.9 & 2.2 & 4.8 & 3.9 \\
\hline Gentamicin & 28.6 & 29.2 & 21.4 & 25.2 & 36.4 & 30.1 \\
\hline Tobramycin & 21.4 & 18.7 & 18.2 & 15.8 & 31.4 & 20.8 \\
\hline Ciprofloxacin & 15.4 & 11.7 & 15.7 & 25.2 & 34.8 & 22.6 \\
\hline Levofloxacin & 15.4 & 11.7 & 15.7 & 24.1 & 31.9 & 20.7 \\
\hline Compound sulfamethoxazole & 35.6 & 38.0 & 44.6 & 40.9 & 44.2 & 42.2 \\
\hline
\end{tabular}

Table 4: Drug resistance rates of ESBL-producing and non ESBL-producing Klebsiella pneumoniae to common antibiotics for 5 consecutive years (\%)

\begin{tabular}{|c|c|c|c|c|c|c|c|c|c|c|}
\hline \multirow{2}{*}{ Antibiotics } & \multicolumn{2}{|c|}{$2012(n=235)$} & \multicolumn{2}{|c|}{$2013(n=291)$} & \multicolumn{2}{|c|}{$2014(n=225)$} & \multicolumn{2}{|c|}{$2015(n=234)$} & \multicolumn{2}{|c|}{$2016(n=207)$} \\
\hline & $\begin{array}{l}\text { ESBLs }(+) \\
(\mathbf{n}=76)\end{array}$ & $\begin{array}{l}\text { ESBLs (-) } \\
(\mathrm{n}=159)\end{array}$ & $\begin{array}{l}\text { ESBLs }(+) \\
(\mathbf{n}=\mathbf{8 5})\end{array}$ & $\begin{array}{l}\text { ESBLs }(-) \\
(\mathrm{n}=206)\end{array}$ & $\begin{array}{l}\text { ESBLs }(+) \\
(\mathbf{n}=95)\end{array}$ & $\begin{array}{l}\text { ESBLs }(-) \\
(\mathbf{n}=130)\end{array}$ & $\begin{array}{l}\text { ESBLs }(+) \\
(\mathbf{n}=102)\end{array}$ & $\begin{array}{l}\text { ESBLs (-) } \\
(\mathrm{n}=132)\end{array}$ & $\begin{array}{l}\text { ESBLs (+) } \\
(\mathbf{n}=90)\end{array}$ & $\begin{array}{l}\text { ESBLs (-) } \\
(\mathrm{n}=117)\end{array}$ \\
\hline Piperacillin & 100 & 6.4 & 100.0 & 3.4 & 100.0 & 4.6 & 99.0 & 5.3 & 100 & 5.1 \\
\hline $\begin{array}{l}\text { Piperacillin/ } \\
\text { Tazobactam }\end{array}$ & 2.6 & 0.0 & 9.4 & 0.5 & 2.1 & 0.0 & 2.9 & 0.0 & 15.6 & 0.0 \\
\hline Cefazolin & 100 & 16.1 & 100 & 14.6 & 100 & 7.8 & 100 & 1.5 & 100 & 12.1 \\
\hline Cefuroxime & 100 & 8.4 & 100 & 10.3 & 100 & 3.8 & 100 & 0.0 & 100 & 8.5 \\
\hline Ceftazidime & 50.0 & 0.0 & 42.4 & 0.0 & 31.6 & 0.0 & 25.5 & 0.0 & 54.4 & 0.0 \\
\hline Ceftriaxone & 100 & 0.0 & 100 & 0.0 & 100 & 0.0 & 100 & 0.0 & 100 & 0.0 \\
\hline Cefotaxime & 100 & 0.0 & 100 & 0.0 & 100 & 0.0 & 100 & 0.0 & 100 & 0.0 \\
\hline Cefepime & 48.7 & 0.0 & 40.0 & 0.0 & 57.4 & 0.0 & 59.2 & 0.0 & 54.4 & 13.7 \\
\hline Cefoxitin & 24.0 & 2.7 & 27.0 & 2.5 & 16.0 & 0.0 & 5.9 & 1.5 & 4.4 & 4.3 \\
\hline Aztreonam & 48.7 & 0.0 & 38.8 & 0.0 & 35.1 & 0.0 & 31.4 & 0.0 & 77.8 & 4.3 \\
\hline Imipenem & 0.0 & 0.0 & 0.0 & 0.0 & 1.1 & 0.0 & 0.0 & 0.0 & 6.7 & 0.0 \\
\hline Meropenem & 0.0 & 0.0 & 0.0 & 0.0 & 1.1 & 0.0 & 0.0 & 0.0 & 6.7 & 0.0 \\
\hline Amikacin & 11.8 & 0.6 & 7.1 & 1.0 & 1.1 & 0.8 & 2.9 & 1.5 & 10.0 & 0.9 \\
\hline Gentamicin & 69.7 & 10.3 & 74.9 & 10.7 & 44.7 & 5.4 & 44.1 & 10.6 & 74.4 & 6.9 \\
\hline Tobramycin & 53.4 & 5.8 & 52.9 & 4.4 & 40.0 & 0.0 & 31.4 & 3.8 & 67.8 & 1.7 \\
\hline Ciprofloxacin & 40.8 & 3.2 & 27.1 & 5.3 & 32.0 & 0.0 & 55.0 & 6.8 & 76.6 & 5.1 \\
\hline Levofloxacin & 40.8 & 2.6 & 27.1 & 5.3 & 32.0 & 0.0 & 55.0 & 7.6 & 73.3 & 5.1 \\
\hline $\begin{array}{l}\text { Compound } \\
\text { sulfamethoxazole }\end{array}$ & 90.0 & 12.9 & 93.9 & 16.9 & 88.1 & 0.0 & 80.0 & 11.2 & 94.4 & 8.5 \\
\hline
\end{tabular}


The investigation data showed that the detection rate of ESBL-producing Klebsiella pneumoniae was $37.6 \%$ on average, which was consistent with relevant literature reports. ${ }^{[7-9]}$ The detection rates of ESBL-producing Klebsiella pneumoniae for 5 consecutive years were $32.3 \%$, $29.2 \%, 42.2 \%, 43.6 \%$ and $43.5 \%$ respectively, showing an increasing trend year by year, and they were higher than the national average values published by CARSS in the same period. It may be related to the insufficient environmental disinfection and hand hygiene, which may cause crossinfection and increase the infection rate. ${ }^{[10]}$ Therefore, it is necessary to take effective disinfection and isolation measures aimed at nosocomial infection to prevent Klebsiella pneumoniae from being prevalent in the hospital as far as possible.

As is shown in Table 3, the drug resistance rates of Klebsiella pneumoniae to piperacillin, cephalosporins (excluding ceftaxidine and cefepime) and compound sulfamethoxazole are all more than $40 \%$, suggesting that Klebsiella pneumoniae is severely resistant to the above drugs, which should not be used as empirical drugs for Klebsiella pneumoniae infection clinically. The data in Table 4 show that, the drug resistance rates of the ESBL-producing strains to the antibiotics in the drug susceptibility spectrum (except imipenem, meropenem and amikacin) are significantly higher than those of non ESBL-producing strains. In addition, the drug resistance rates of ESBL-producing Klebsiella pneumoniae to piperacillin, all cephalosporins (except for cephamycins), gentamicin, tobramycin and compound sulfamethoxazole exceed $40 \%$, and the drug resis-

\section{References}

[1] Hu ZJ, Pan XL, Zhou DS, et al. Clinical distribution and surveillance of bacterial resistance of Klebsiella pneumoniae. Chinese Journal of Nosocomiology. 2014; 24(12): 2865-2867.

[2] Cui C, Zhu WM, Tang XH. Surveillance on antimicrobial resistance in clinical isolates of Klebsiella pneumoniae from 2010 to 2013. Chinese Journal of Antibiotics. 2015; 40(5): 372-381.

[3] He SN, Chen M, Jin HY. Analysis of clinical distribution and drug resistance of Klebsiella pneumoniae. Chinese Journal of Nosocomiology. 2015; 25(19): 4345-4346, 4355.

[4] Zhang Q, Liu QQ. Analysis on TCM clinical characteristics of Klebsiella pneumoniae sepsis. Beijing: Beijing University of Chinese Medicine; 2014

[5] Qiu GC, Sun MZ, Shao LR. The distribution and drug resistance analysis of Klebsiella pneumoniae in hospital environment. Laboratory Medicine. 2015; 30(2): 160-162, 166. tance rates to cefoxitin, carbapenems and enzyme inhibitors are very low. The important resistance mechanism of Klebsiella pneumoniae to $\beta$-lactams may be the production of plasmid-mediated ESBLs, which can hydrolyze penicillin, cephalosporins and $\beta$-lactamases of monocyclic $\beta$-lactams and are only susceptible to cephamycins, carbapenems and enzyme inhibitors. The reason for the high resistance of ESBL-producing strains to aminoglycosides and sulfonamides may be related to the fact that the ESBL plasmids carried by the enzyme-producing strains can also carry corresponding resistance genes at the same time, which makes ESBL-producing strains simultaneously resistant to a variety of antibiotics with the results of multiple drug resistance. Meanwhile, four strains are found to be totally resistant to antibiotics, and the corresponding mechanism remains to be further studied.

To sum up, in terms of department and specimen distribution, the isolated strains of Klebsiella pneumoniae are mainly from Respiratory Medicine Department and lower respiratory tract. It can be seen that Klebsiella pneumoniae is one of the main pathogens causing pulmonary infection. Clinicians must establish effective treatment options, rationally select antibiotics on the basis of the production of ESBLs and DST results, change the habit of clinical medication to effectively control the occurrence of Klebsiella pneumoniae infection.

\section{Conflicts of Interest Disclosure}

The authors have no conflicts of interest related to this article.

[6] Zhou R, Zhu WM, Huang WX. Distribution and antibiotic resistance of 855 isolates of Klebsiella pneumoniae. Chinese Journal of Antibiotics. 2013; 38(5): 363-369.

[7] Li HJ, Zhou DP, Zhao N. Clinical distribution and change of drug resistance of Klebsiella pneumoniae during 2011-2013. Chinese Journal of Nosocomiology. 2015; 25(12): 2670-2672.

[8] Zou ZY, Liu SQ, Wang Q, et al. Study on drug resistance characteristics of Klebsiella pneumoniae separated from different specimens sources. Laboratory Medicine and Clinic. 2015; 12(22): 3300-3303.

[9] Hu FP, Zhu DM, Wang F. CHINET 2013 surveillance of bacterial resistance in China. Chinese Journal of Infection and Chemotherapy. 2014; 14(5): 365-374.

[10] Brust K, EvansA, Plemmons R. Tigecycline in treatment of multidrug-resistant Gram-negative bacillus urinary tract infections: a systematic review. J Antimicrob Chemother. 2014; 69(10): 2606-2610. PMid: 24879669. https://doi.org/10.1093/jac/ dku189 